\title{
A Hollow Cathode Magnetron (HCM)
}

\author{
Zhehui Wang and Samuel A. Cohen \\ Plasma Physics Laboratory, Princeton University, P.O. Box 451, Princeton, NJ 08543
}

(April 16,1998)

\begin{abstract}
A new type of plasma sputtering device, named the hollow cathode magnetron (HCM), has been developed by surrounding a planar magnetron cathode with a hollow cathode structure (HCS). Operating characteristics of HCMs, current-voltage $(I-V)$ curves for fixed discharge pressure and voltagepressure $(V-p)$ curves for fixed cathode current, are measured. Such characteristics are compared with their planar magnetron counterparts. New operation regimes, such as substantially lower pressures $(0.3$ mTorr $)$, were discovered for HCMs. Cathode erosion profiles show marked improvement over planar magnetron in terms of material utilization. The use of HCMs for thin film deposition are discussed.
\end{abstract}

PACS numbers: 51.50.+v, 52.50.Dg, 52.75.Rx 


\section{INTRODUCTION}

Planar sputtering magnetrons, FIG. 1, are important tools for thin film deposition and ion etching [1]. With some modification, planar magnetrons also have potential to become high intensity hyperthermal atomic beam sources [2]. Despite their technological importance and potential in industry, planar sputtering magnetrons also have some limitations.

Relatively high gas pressures are required for planar magnetron discharges. Conventional planar magnetrons are usually operated at pressures greater than $1 \mathrm{~m}$ Torr. Although these pressures are substantially lower than in a diode discharge, they are still high enough that gas incorporation could affect the deposited thin film quality. Also at these pressures, the mean-free-paths of sputtered particles are only a few centimeters. These particles are easily scattered and deposit on places, such as vacuum windows, that are close to the cathode. The scattering of sputtered atoms by ambient gas atoms and molecules effectively reduces useful flux to the substrate. Collisions of sputtered atoms with ambient gases also heats the gas. The increase in gas temperature reduces the gas density close to the target surface, which changes the operational characteristics in a complicated way [3]. One method to extend magnetron operation into sub-mT regime, as invented by Cuomo and Rossnagel [4], was the introduction of an electron gun to form the so-called triode configuration. The electron gun can provide several amperes of energetic electron current, which sustains magnetron discharge at pressure near $0.1 \mathrm{mT}$.

Low target-material utilization may occur because of the concentration of plasma in certain configurations. The plasma is concentrated by the magnetic and the electric fields in a limited area of the total target surface. This leads to a trench formation of 'race track' shape as more material is sputtered here than anywhere else on the target. The target, therefore, gets sputtered-through at the trench bottom while the rest of the target surface is less eroded. Typically, in a conventional magnetron one expects utilization of $25-30 \%$

of the total target material [5], [6]. Currently there are designs available where the target and/or the magnets are rotated in order to keep the trench moving from place to place on 
the target to achieve a higher target utilization [7] [8] [9]. Target utilization in magnetron sputtering can also be enhanced by flattening the magnetic field lines parallel to the target surface [10] [11] [12].

Other improvements desirable in conventional planar magnetron thin film deposition include directionality control [13] [14] and uniformity of the film deposited.

We have approached the problems through modification of the cathode geometry of the conventional planar sputtering magnetrons by adding a HCS.

\section{EXPERIMENT}

The experiments were carried out in a cylindrical, stainless steel vacuum chamber 55.9 $\mathrm{cm}$ in diameter and about $134.6 \mathrm{~cm}$ in length. The magnetron target was mounted offcenter at one end of the vacuum chamber. A diffusion pump of $1500 \mathrm{l} / \mathrm{sec}$ backed by a mechanical pump evacuated the chamber into a base pressure of less than $1 \times 10^{-6}$ Torr. An ionization gauge, with three emission current scales, measured the pressure below $10 \mathrm{mT}$; a thermocouple gauge measured the pressure above 2 mTorr; and a capacitance manometer (Baratron) covered the pressure range from 1 Torr to about $0.1 \mathrm{~m}$ Torr. These gauges were mounted on the side wall of the vacuum chamber and placed about $63.5 \mathrm{~cm}$ away from the pump to ensure an accurate reading of the chamber pressure. For most of the experiments, a gate valve between the vacuum chamber and the diffusion pump was not fully open. Through adjustment of the opening of the gate valve, the steady state pressure was regulated. Highpurity grade gases (either $\mathrm{Ar}$ or $\mathrm{N}_{2}$ ) were introduced into the chamber from the same end of the vacuum chamber as the target, at about $20 \mathrm{~cm}$ from the cathode. Two kinds of gas could be introduced into the experimental chamber at once. The flow rate of the gases were measured by Sierra Top $=$ Trak digital flow meters. The flow rate were usually in the range of 0.5 to $10 \mathrm{sccm}$. Precision needle valves were used to control the flow. The steady state pressure was reached when the pumping rate was balanced by the gas feed rate.

A Hipotronics DC power supply, which can output up to $400 \mathrm{~mA}$ at $3 \mathrm{kV}$, was used to 
bias the target to a negative potential relative to the vessel wall. A ballast resistor of $5 \mathrm{k} \Omega$ was connected in series with the magnetron cathode to limit the current flow through the system. The power supply was operated in a constant voltage mode. Through adjustment of the output voltage, the discharge current was regulated.

The dimensions of the planar magnetron cathode (also called target in this paper), FIG. 2, were $11.4 \mathrm{~cm}$ in diameter, and about $0.2 \mathrm{~cm}$ in thickness. Most of time, the target was made of $\mathrm{Cu}$ for $\mathrm{HCM}$ characteristic studies. Energetic neutral particle reflection measurements were also carried out and will be described in another paper. The magnetron target was cooled by room temperature de-ionized water to prevent target melting in high power operation. Ceramic permanent magnets were used to produce the semi-dipole-shape magnetic field configuration.

A thin metallic HCS with length $L$ and diameter $D$, defined in FIG. 2, was mechanically attached to a planar magnetron cathode to modify the planar magnetron into a HCM. HCSs were made of stainless steel for this study. The thickness of HCSs ranges from less than 0.1 to $0.4 \mathrm{~cm}$. The mounting of a HCS can be quite flexible. A HCS can either be electrically connected to the target, or electrically insulated from the target, and the HCS electric potential can be controlled separately from the cathde (This permitted measurements of current flow to the HCS).

\section{RESULTS}

Voltage as a function of pressure $(V-p)$ was measured for pure $\operatorname{Ar}$ and $\mathrm{N}_{2}$ plasmas separately at a fixed total cathode current of $300 \mathrm{~mA}$ for a HCM with different $L$ s and $D=$ $12 \mathrm{~cm}$, (FIG. 3) and (FIG. 4). Planar magnetron results under similar conditions are shown for comparison. Several features of HCM are most obvious.

HCMs can be maintained at substantially lower pressure than planar sputtering magnetrons under similar conditions. The exact value of the lowest pressure depends on the gas species, magnetic field strength, discharge current, and the target material. In our magnetic 
field of about 170 gauss maximum on the target surface, the stable operable pressure for Ar was about $0.30 \mathrm{~m}$ Torr for HCM discharges. For nitrogen, this value was higher at 0.51 mTorr. For the same cathode bias, HCM sustained a lower gas pressure plasma. Or for the same gas pressure, HCM discharges were sustained with lower cathode bias. These were the evidences for more efficient use of electron energy, or improvement of energetic electron confinement.

For other conducting target materials, similar sub-mT $V-p$ operation regimes were also observed. These targets included carbon ( $Z=6$, FIG. 5), iron ( $Z=26$, FIG. 9$)$, molybdenum $(Z=42$, FIG. 7 ), tantalum $(Z=72$, FIG. 8$)$, and tungsten $(Z=73$, FIG. 6 ) .

$I-V$ curves were measured for $\mathrm{Ar}$ and $\mathrm{N}_{2}$ discharges at a wide range of pressures, Fig. (10) and Fig. (11). In general, we saw the dependence of current on the voltage is stronger at high pressures than at low pressure regime.

Comparison of $I-V$ characteristics of HCM with planar magnetron is made in FIG. 12 for a pressure of about $5 \mathrm{~m}$ Torr. Again we see that for the same cathode current, the HCM discharge can be sustained at a lower cathode bias. Conversely, for the same cathode bias, the total HCM cathode current is greater than the planar magnetron current. This was generally observed for higher pressures, when comparison was possible. For low pressure discharges, the HCM discharges had no planar magnetron counterpart.

The distribution of total cathode current $\left(I_{t o t}\right)$ between the planar magnetron cathode base $\left(I_{\text {target }}\right)$ and the HCS $\left(I_{H C S}\right)$, satisfying $I_{\text {tot }}=I_{\text {target }}+I_{H C S}$, was also studied. Results of the current distribution is shown in FIG. 13.

Two regimes of the cathode current distribution were evident. For the high-pressure regime $(p>7$ mTorr $)$, the HCS received about $15 \%$ of the total cathode current. For $p$ $<7$ mTorr, the HCS current increased with decreasing in gas pressure, until the discharge disappeared, at which, about $30 \%$ of the total cathode current went to the side wall. The trend of HCS current increasing with decreasing gas pressure was an indication of plasma expansion away from the target. This was confirmed by visual examination of the plasma. 
Similar expansion of plasma has been observed on un-magnetized hollow cathode discharges at low pressures. In-between these two pressure regime, a HCS current minimum existed.

The current distribution measurement as described above showed that the HCS current was not negligible, particularly in the new found low-pressure regime. However, the majority of the cathode current flowed to the target. The ion sputtering of the target was intensive. This was verified by the observation of net deposition on the HCS wall, while the bottom plate was sputter eroded.

Target erosion in HCM, as well as planar magnetrons, is due to the sputtering of the target surface by energetic ions. These ions sputter target atoms off. What left behind is the erosion profile. For planar magnetrons, the erosion mostly took place where the B field was parallel to the cathode surface. This causes the well-known phenomenon of trench formation in a 'race track' shape. For HCM operation at high pressures, when the glow architecture was similar to the planar magnetron case, trench formation was also observed. However, at low pressures (less than 4 mTorr), much larger area received net ion sputtering in HCMs.

When we operated our planar magnetron without a HCS, a trench was formed at a radius of $1.6 \mathrm{~cm}$. The total trench area was about $3.2 \mathrm{~cm}^{2}$. The area received much less sputtering was larger than the trench area, $17.7 \mathrm{~cm}^{2}$. In comparison, for HCM operations at pressures less than $4 \mathrm{mT}$, with $\mathrm{W}, \mathrm{Al}$ and $\mathrm{C}$ targets, the ion-sputtered areas were a concentric ring zone that had an inner radius of about $1.27 \mathrm{~cm}$ and an outer radius $4.76 \mathrm{~cm}$ (only limited by the inner wall of the HCS). The total area of such concentric zone was measured to be about $66 \mathrm{~cm}^{2}$. A 90-hour C-target erosion for Ar discharge at $3 \mathrm{~m}$ Torr and 300-m A cathode current showed that a deep trench was formed all across the $66-\mathrm{cm}^{2}$ ring zone.

The erosion study showed that HCM low pressure operation can substantially increase the utilization of the target material. More than $80 \%$ of the exposed target surfaces were ion sputter eroded in HCMs studied. 


\section{DISCUSSION}

To fit the data points as shown in FIG. 5 to FIG. 9, cathode biases were related to the gas pressure through an empirical function

$$
V=U_{0}+\frac{\kappa_{1}}{p^{m}}+\kappa_{2} p
$$

Optimal values of the parameters $U_{0}, \kappa_{1}, \kappa_{2}, m$ were found using IDL curvefit.pro algorithm. Typical values for these parameters are $U_{0}=300$ to $600, \kappa_{1}=20$ to $300, \kappa_{2}=-1$ to -7 and $m=0.5$ to 3.0. Such fitting are shown as solid curves in FIG. 5 to FIG. 9.

A 0-D steady-state discharge model was developed from ion density balance and power balance [15]. It was found that cathode bias $V$ to sustain the discharge is determined by

$$
V=\frac{\xi}{\gamma g}+\frac{\langle E\rangle}{\tau_{e} \nu_{i} \gamma g}
$$

Where $\xi$ is the energy required to creat one ion-electron pair. $\xi$ is about $30 \mathrm{eV}$ for $\mathrm{Ar}$ magnetron discharge [16]. $g$, satisfying $0<g<1$, is a factor determined by system geometry, $\gamma$ is the secondary electron emission coefficient, $\langle E\rangle$ is the average energy loss for electrons reaching anode, $V$ is the volume of the discharge, $\tau_{e}$ is average electron confinement time in the system, $\nu_{i}$ is the electron ionization collision frequency.

Compare Eq. (1) to Eq. (2), we conclude that

$$
U_{0}=\frac{\xi}{\gamma g}
$$

As an estimation, for $\gamma=0.1$ and $g=0.5, U_{0}=600$. This $U_{0}$ number matches well with the fitting value of $U_{0}=300$ to 600 .

The second term in Eq. (2) describes electron energy loss in a magnetron discharge. The electron energy loss is pressure dependent due to collisions of electrons with gas particles. The electron energy loss is also dependent upon electron electron collisions and other effects. In the high pressure end, we speculate the energy loss is proportional to pressure, while in the low pressure end, the electron energy loss is inversely proportional to pressure to certain power. Such considerations are described by the second and third term in our fit Eq. (1). 
A commonly used empirical law of $I$ dependence on cathode bias $V$ in the planar magnetron case is

$$
I=k V^{n}
$$

The exponent $n$ is usually in the range of 2 to 20. This law still holds for HCM discharges at high pressures. However, in the low pressure regime, a modification into the form

$$
I=k\left(V-V_{0}\right)^{n}
$$

provides a much better fit, FIG. 15 .

The exponent $n$ as defined in Eq. (4) and Eq. (5) depends on the discharge pressure among other factors (such as geometry, target material, magnetic field, surface condition).

The differences between a HCM and a planar magnetron operation can be attributed to hollow cathode effects. A planar sputtering magnetron is a very good confinement system for energetic electrons that have energies above the ionization threshold. So in a sense, adding a HCS to a planar sputtering magnetron is like gilding the lily, improving the utilization of electron energy by about $20 \%$ from our $I-V$ comparison curve, Fig. 12.

Geometry of a HCS can affect many aspects of the planar sputtering magnetron operation through several processes. These processes jointly cause the net hollow cathode effects. Obviously, a HCS is most important to primary electrons and other electrons (with energies above the ionization threshold) that would have been lost without a HCS. Hollow cathode effects include more efficient use of electron energy through increasing ionization path length of electrons in the system; more efficient use of ions and uv photons which can generate more secondary electrons from HCS; etc. Quantitative description of hollow cathode effects will be left for future works.

Larger target area receives sputtering in a HCM than in a planar sputtering magnetron, therefore higher utilization of target material is expected. This is consistent with the visual inspection of the glow structure difference in two systems. In a HCM, plasma glow extends to a much larger area than a planar magnetron due to HCS. Larger plasma area causes more 
sputtering in a HCM than in a planar magnetron. Better utilization of target material is highly desirable for thin film deposition applications.

\section{SUMMARY AND CONCLUSION}

With addition of a hollow cathode structure (HCS) to a conventional planar sputtering magnetron, we have developed a new type of sputtering magnetron device, hollow cathode magnetron (HCM). This name was chosen over the name of a "magnetized hollow cathode' (MHC) because many properties of such a device are extensions of planar magnetron behavior.

In terms of $V-p$ curves at constant cathode current, HCMs were found to be operable at substantially lower pressures. In particular, for $\mathrm{Ar}$ discharge with $\mathrm{Cu}$ cathode, a 0.3 mTorr stable operation was possible at a cathode current of $300 \mathrm{~mA}$. This pressure value can be compared with the usual planar magnetron lowest pressure of about $1-2$ mTorr. Operation at lower gas pressures is desirable for thin film deposition because there would be less scattering of sputtered particles and less collisions for energetic particles. Therefore deposition particle flux and energy flux can be increased. Gas incorporation into the film can also be reduced. Thin film quality can be modified.

In terms of $I-V$ curves, the usual law of $I=k V^{n}$ for planar magnetrons can be used to fit data for high pressures $(p>5 \mathrm{mT})$. For low pressures $(p<2 \mathrm{mT})$, a modified law of $I=k\left(V-V_{0}\right)^{n}$ has been proposed. The exponent are found to be in the range of 1 to 10. The transition is probably is an indication of the hollow cathode effects.

HCM discharge glow structure and target erosion profile studies showed that a HCM target erosion can take place on a much larger surface area than its planar magnetron counterpart. Therefore the well-known trench formation in a shape of 'race track' for conventional planar magnetron can be avoided. Utilization of target material can be improved dramatically. 


\section{ACKNOWLEDGMENT}

This Work is supported by U.S. D.o.E Contract No.DE-AC02-76-CHO-3073 


\section{REFERENCES}

[1] D. A. Glocker and S. Ismat Shah, Handbook of Thin Film Process Technology, Institute of Physics Publishing, Bristol and Philadelphia (1995)

[2] Goeckner, M. J., T. K. Bennett, and S. A. Cohen, Appl. Phys. Lett. 71, 980 (1997)

[3] S. M. Rossnagel, J. J. Cuomo, and W. D. Westwood, Handbook of Plasma Processing Technology, Noyes Publications, NJ (1990)

[4] J. J.Cuomo and S. M. Rossnagel, J. Vac. Sci. Technol. A 4, 393, (1986)

[5] Chapin, J. S., Res. Dev. 25, 37 (1974)

[6] Van Vorous, T., Solid State Technology 19, 62 (1976)

[7] Waits, R. K., J. Vac. Sci. Technol. 15, 179 (1978)

[8] Schiller, S., Heisig, U., and Steinfelder, K., Thin Solid Films 33, 331 (1976)

[9] Hosokawa, N., Tsukada, T. and Misumi, T., J. Vac. Sci. Technol. 14, 143 (1977)

[10] Hata, T. and Kamide, Y., J. Vac. Sci. Technol. A 52154 (1978)

[11] Welty, R. P., US Patent 4,865,798 (1989)

[12] Manlley, B. W., U. S. Patent 5,262,028 (1993)

[13] Rossnagel, S. M. and Mikalsen, D., JVST A9, 261 (1991)

[14] Hayden, D. B., D. R. Juliano, K. M. Green, D. N. Ruzic, C. A. Weiss, K. A. Ashtiani and T. L. Kicata, J. Vac. Sci. Technol. A 16, 4, (1998)

[15] Wang, Z., PH. D thesis, Princeton University (1998)

[16] Lieberman, M. A. and Lichtenberg, A. J., Principles of Plasma Discharge and Material Processing, John Wiley and Sons (1994) 


\section{FIGURES}

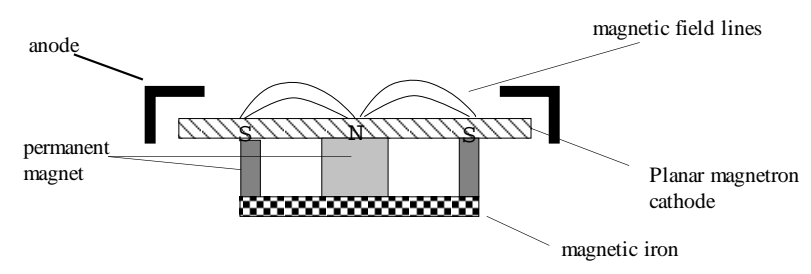

FIG. 1. A schematic of a planar magnetron configuration.

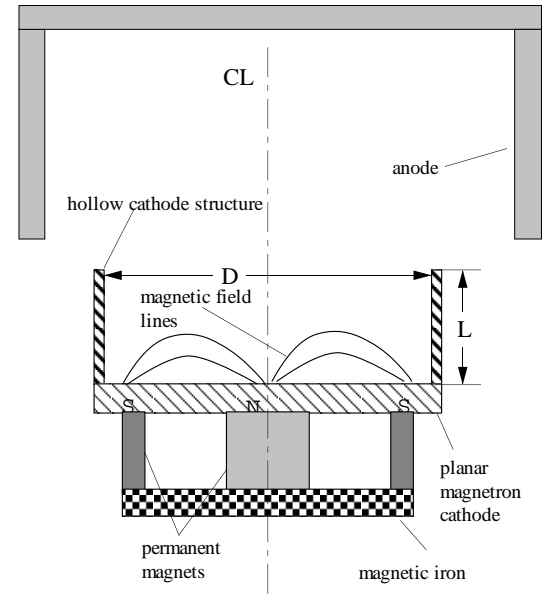

FIG. 2. A HCM configuration with definition of cathode length $L$ and diameter $D$. 


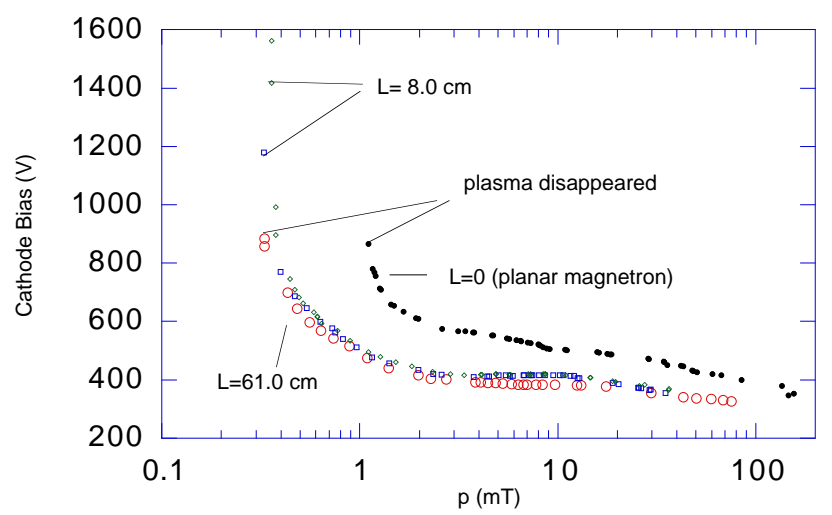

FIG. 3. $V-p$ characteristics for Ar discharge, with $\mathrm{Cu}$ cathode. Total cathode current was 300 mA.

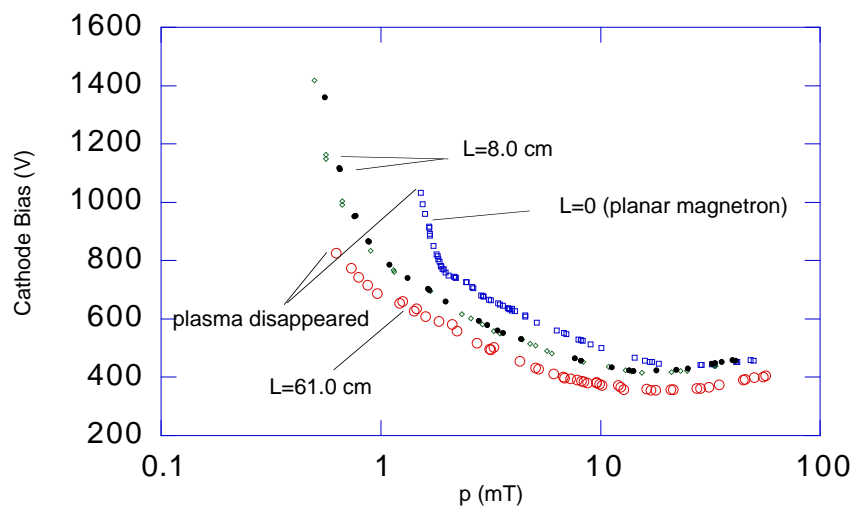

FIG. 4. V-p characteristics for $\mathrm{N}_{2}$ discharge, with $\mathrm{Cu}$ cathode. Total cathode current was 300 mA.

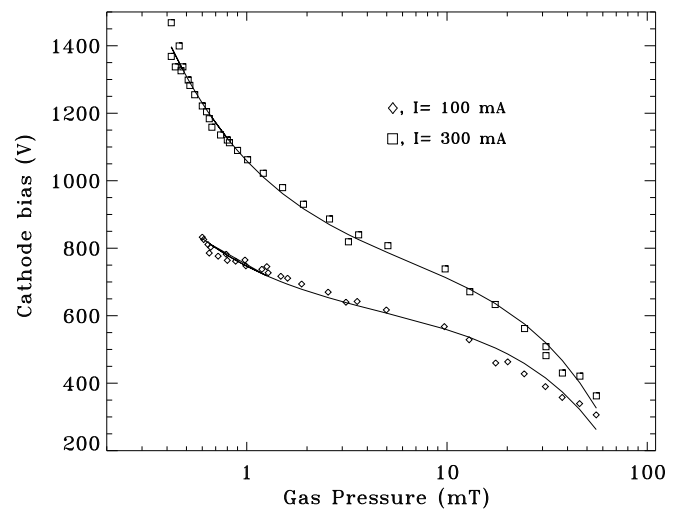

FIG. 5. $V-p$ characteristics for Ar discharge, with carbon cathode. Data points are shown. The solid lines are fits to Eq. (1). 


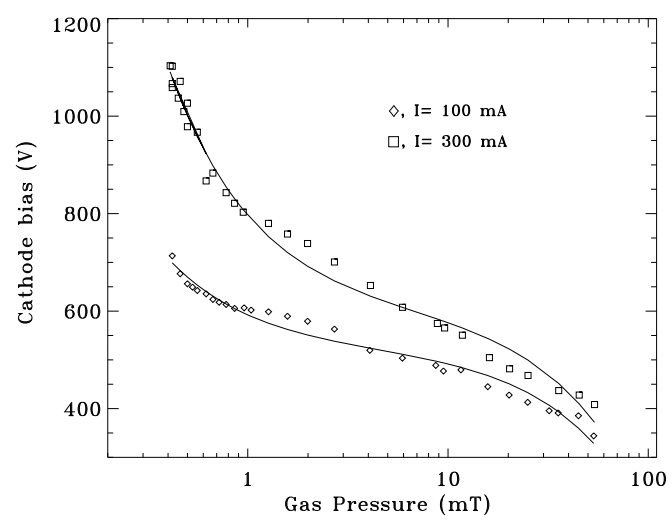

FIG. 6. $V-p$ characteristics for Ar discharge, with Tungsten cathode. Data points are shown. The solid lines are fits to Eq. (1).

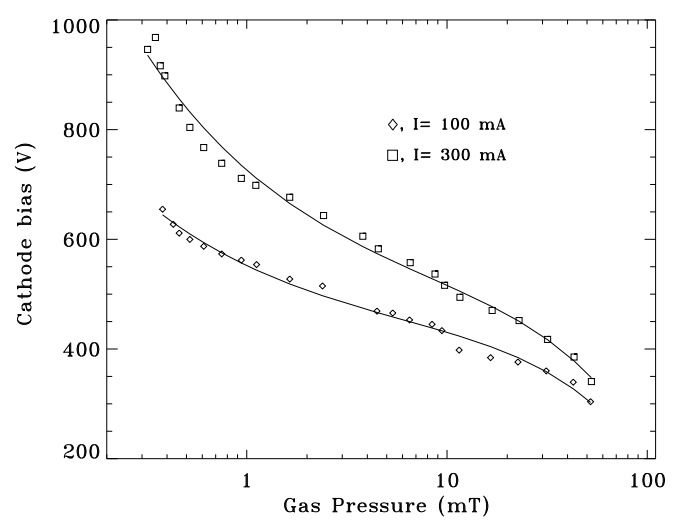

FIG. 7. $V-p$ characteristics for Ar discharge, with Molybdenum cathode. Data points are shown. The solid lines are fits to Eq. (1).

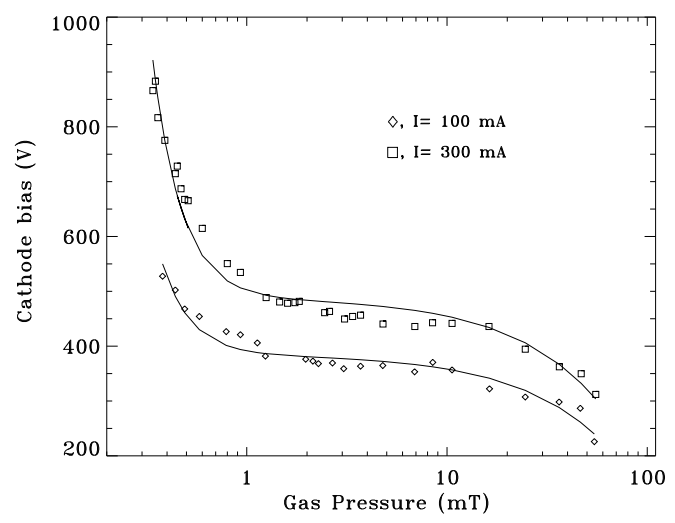

FIG. 8. $V-p$ characteristics for Ar discharge, with Tantalum cathode. Data points are shown. The solid lines are fits to Eq. (1). 


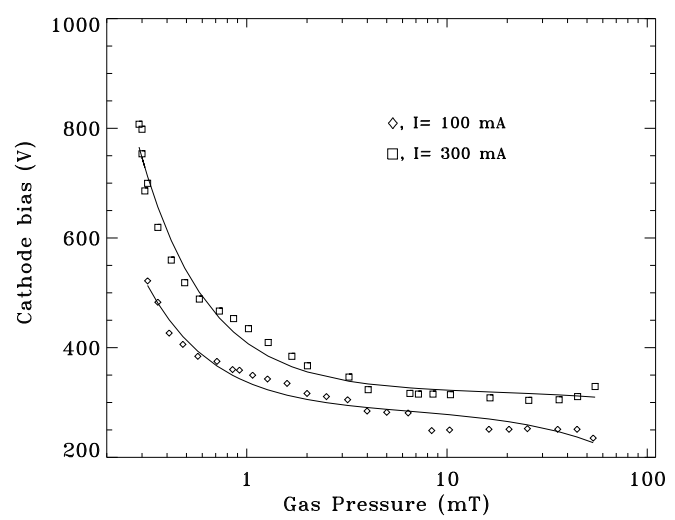

FIG. 9. $V-p$ characteristics for Ar discharge, with Iron cathode. Data points are shown. The solid lines are fits to Eq. (1).

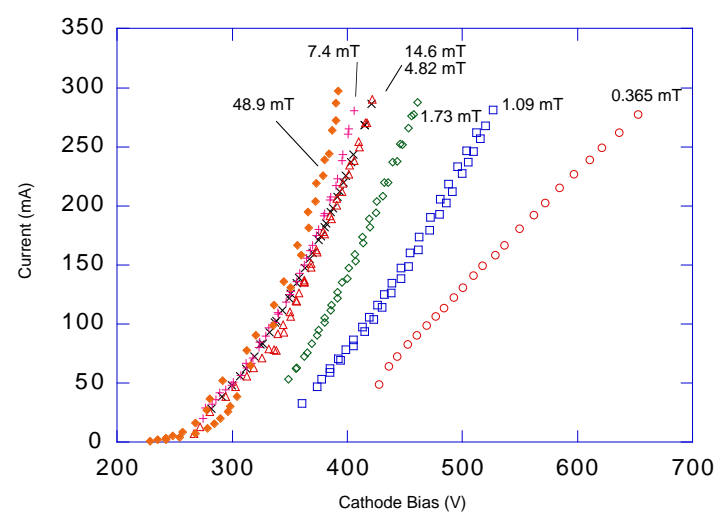

FIG. 10. $I-V$ characteristics for Ar discharge, with $\mathrm{Cu}$ cathode, $\mathrm{L}=61.0 \mathrm{~cm}$. Various pressures were studied.

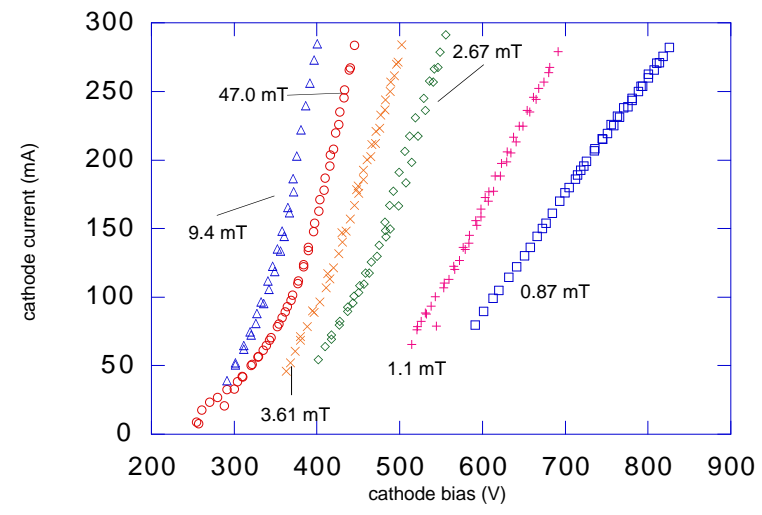

FIG. 11. $I-V$ characteristics for $\mathrm{N}_{2}$ discharge, with $\mathrm{Cu}$ cathode, $\mathrm{L}=61.0 \mathrm{~cm}$. Various pressures were studied. 


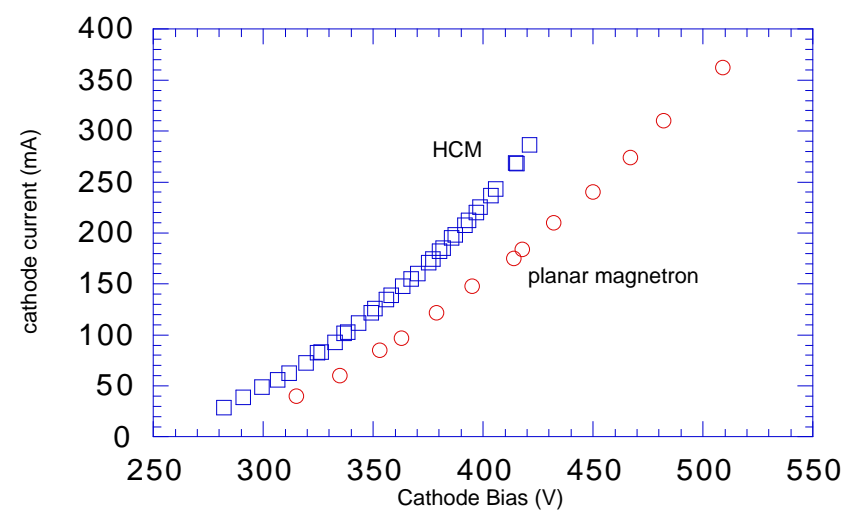

FIG. 12. Comparison of HCM $(\mathrm{L}=61 \mathrm{~cm}) I-V$ characteristics with that of planar magnetrons at about 6 m Torr with $\mathrm{Cu}$ cathode.

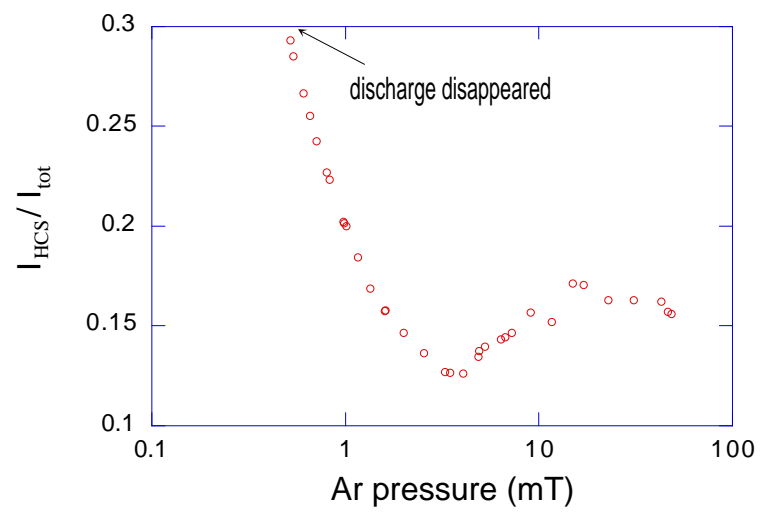

FIG. 13. Measurement of current distribution between HCS and the target for $\mathrm{L}=8.0 \mathrm{~cm}, \mathrm{I}_{\text {tot }}$ $=300 \mathrm{~mA}$, Ar discharge, $\mathrm{Cu}$ target.

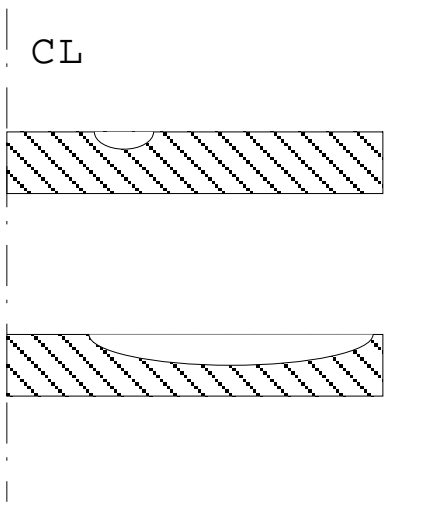

FIG. 14. A schematic of the target erosion profiles for HCM discharge at low and high pressures. 


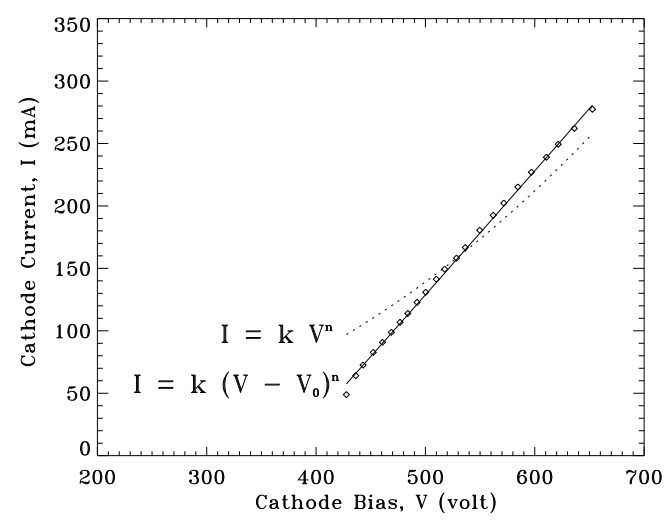

FIG. 15. Comparison of fittings for a HCM $I-V$ curve using different functions.

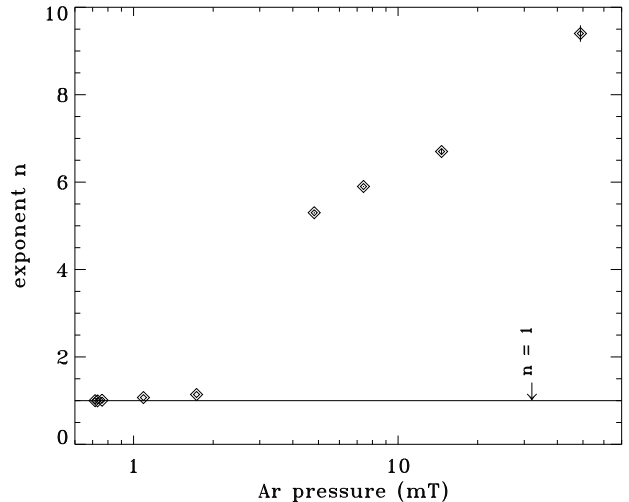

FIG. 16. Exponent $n$ as a function of discharge pressure.

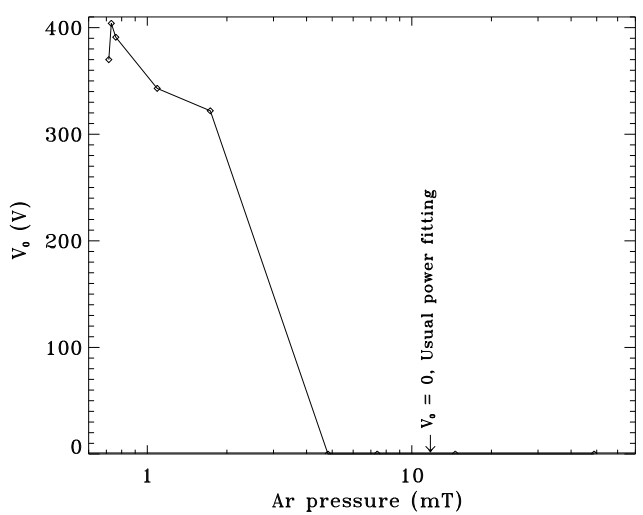

FIG. 17. Bias offset $V_{0}$ as a function of pressure. 